\title{
Pressure-stabilized solvates of xylazine
}

\section{hydrochloride}

\author{
Anna Olejniczak, ${ }^{a}$ Kristine Krūkle-Bērzinga ${ }^{b}$ and Andrzej Katrusiak ${ }^{a *}$
}

${ }^{a}$ Faculty of Chemistry, Adam Mickiewicz University, Umultowska 89b, 61-614 Poznań, Poland

${ }^{b}$ Faculty of Chemistry, University of Latvia, Jelgavas iela 1, LV-1004 Riga, Latvia

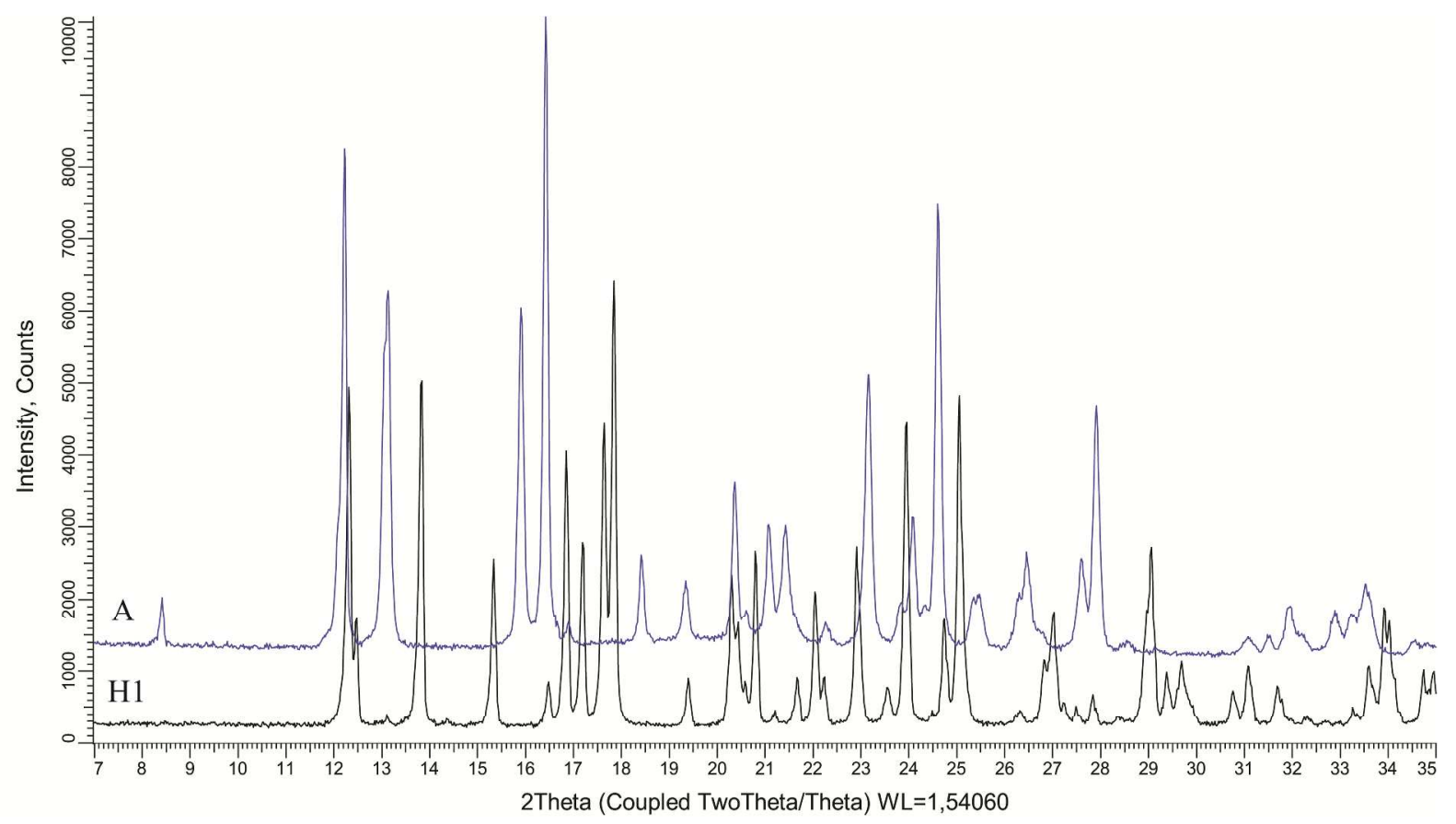

Figure S1. Powder patterns of $\mathrm{XylHCl}$ neat form A and its hydrate H1. 

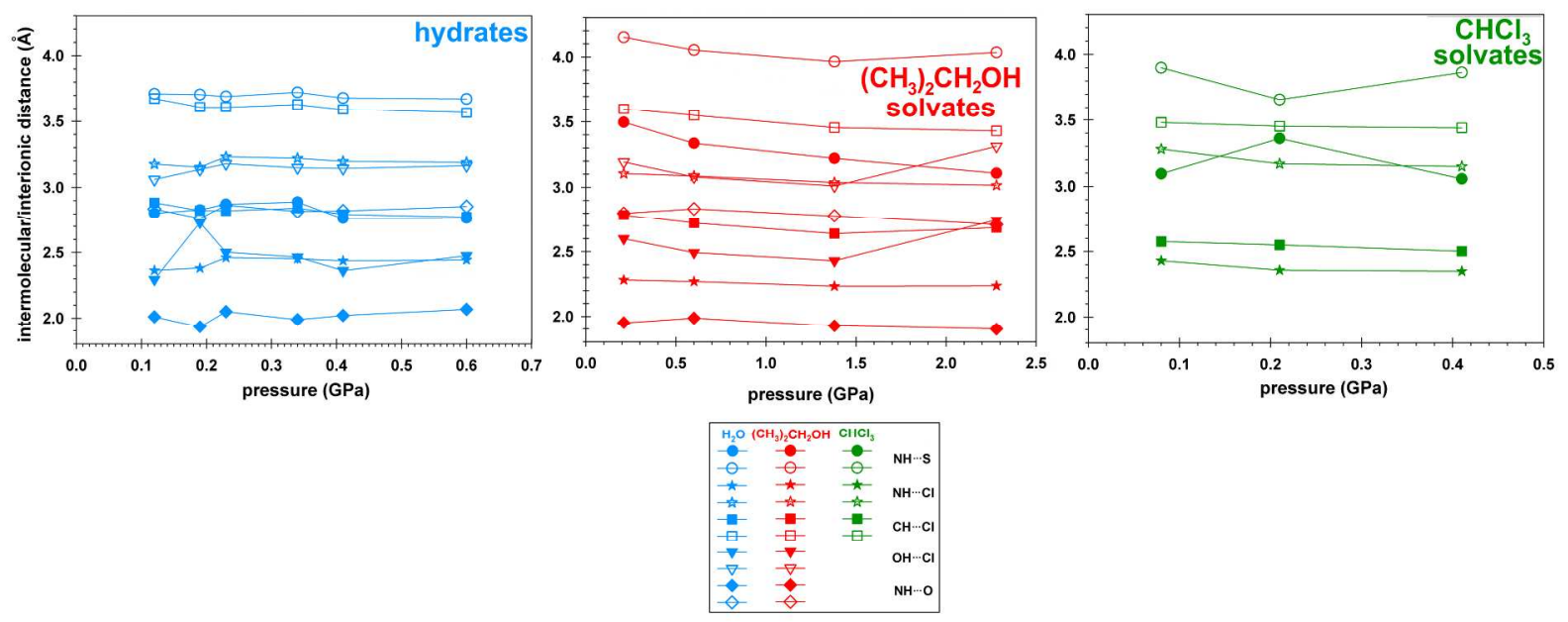

Figure S2. Intermolecular and interionic distances in $\mathrm{XylHCl}$ hydrates, $(\mathrm{CH} 3)_{2} \mathrm{CH}_{2} \mathrm{OH}$ and $\mathrm{CHCl}_{3}$ solvates as a function of pressure.

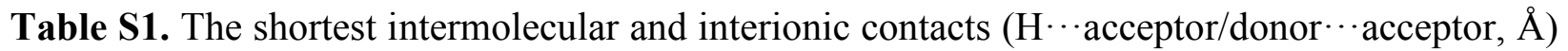
of $\mathrm{XylHCl}$ and its solvates.

\begin{tabular}{|c|c|c|c|c|c|c|}
\hline $\mathrm{XylHCl}$ & $0.11 \mathrm{GPa}$ & & & & & \\
\hline $\mathrm{CH} \cdots \mathrm{S}$ & $\begin{array}{l}3.147(1) / \\
4.144(6)\end{array}$ & & & & & \\
\hline $\mathrm{NH}^{\cdots}{ }^{\cdots \mathrm{Cl}}$ & $\begin{array}{l}2.255(1) / \\
3.124(4)\end{array}$ & & & & & \\
\hline $\mathrm{CH} \cdots \mathrm{Cl}$ & $\begin{array}{l}2.870(1) / \\
3.749(6)\end{array}$ & & & & & \\
\hline $\mathrm{XylHCl} \cdot \mathrm{CH}_{3} \mathrm{Cl}$ & $0.08 \mathrm{GPa}$ & $0.21 \mathrm{GPa}$ & $0.41 \mathrm{GPa}$ & & & \\
\hline $\mathrm{CH} \cdots \mathrm{S}$ & $\begin{array}{l}3.095(3) / \\
3.90(5)\end{array}$ & $\begin{array}{l}3.036(8) / \\
3.66(3)\end{array}$ & $\begin{array}{l}3.056(9) / \\
3.65(10)\end{array}$ & & & \\
\hline $\mathrm{NH}{ }^{\cdots} \mathrm{Cl}$ & $\begin{array}{l}2.429(12) / \\
3.28(4)\end{array}$ & $\begin{array}{l}2.357(15) / \\
3.17(5)\end{array}$ & $\begin{array}{l}2.349(12) / \\
3.15(4)\end{array}$ & & & \\
\hline $\mathrm{CH} \cdots \mathrm{Cl}$ & $\begin{array}{l}2.575(5) / \\
3.48(2)\end{array}$ & $\begin{array}{l}2.549(4) / \\
3.452(19)\end{array}$ & $\begin{array}{l}2.500(3) / \\
3.439(14)\end{array}$ & & & \\
\hline $\mathrm{XylHCl} \cdot\left(\mathrm{CH}_{3}\right)_{2} \cdot \mathrm{CH}_{2} \mathrm{OH}$ & $0.21 \mathrm{GPa}$ & $0.60 \mathrm{GPa}$ & $1.38 \mathrm{GPa}$ & $2.38 \mathrm{GPa}$ & & \\
\hline $\mathrm{CH} \cdots \mathrm{S}$ & $\begin{array}{l}3.497(7) / \\
4.15(3)\end{array}$ & $\begin{array}{l}3.335(2) / \\
4.053(11)\end{array}$ & $\begin{array}{l}3.221(3) / \\
3.65(14)\end{array}$ & $\begin{array}{l}3.108(3) / \\
4.035(3)\end{array}$ & & \\
\hline $\mathrm{NH}{ }^{\cdots} \mathrm{Cl}$ & $\begin{array}{l}2.282(4) / \\
3.105(12)\end{array}$ & $\begin{array}{l}2.270(5) / \\
3.087(16)\end{array}$ & $\begin{array}{l}2.234(5) / \\
3.037(18)\end{array}$ & $\begin{array}{l}2.237(4) / \\
3.015(16)\end{array}$ & & \\
\hline $\mathrm{CH}^{\cdots}{ }^{\cdots} \mathrm{Cl}$ & $\begin{array}{l}2.786(4) / \\
3.60(2)\end{array}$ & $\begin{array}{l}2.722(6) / \\
3.55(3)\end{array}$ & $\begin{array}{l}2.639(7) / \\
3.45(3)\end{array}$ & $\begin{array}{l}2.685(5) / \\
3.43(3)\end{array}$ & & \\
\hline $\mathrm{OH}^{\cdots} \mathrm{Cl}$ & $\begin{array}{l}2.599(14) / \\
3.19(7)\end{array}$ & $\begin{array}{l}2.493(9) / \\
3.08(2)\end{array}$ & $\begin{array}{l}2.429(10) / \\
3.01(4)\end{array}$ & $\begin{array}{l}2.745(11) / \\
3.31(13)\end{array}$ & & \\
\hline $\mathrm{NH} \cdots \mathrm{O}$ & $\begin{array}{l}1.95(2) / \\
2.80(2)\end{array}$ & $\begin{array}{l}1.987(11) / \\
2.836(18)\end{array}$ & $\begin{array}{l}1.928(14) / \\
2.78(2)\end{array}$ & $\begin{array}{l}1.90(3) / \\
2.71(3)\end{array}$ & & \\
\hline $\mathrm{XylHCl} \cdot\left(\mathrm{CH}_{3}\right)_{2} \cdot \mathrm{H}_{2} \mathrm{O}$ & $0.12 \mathrm{GPa}$ & $0.19 \mathrm{GPa}$ & $0.23 \mathrm{GPa}$ & $0.34 \mathrm{GPa}$ & $0.41 \mathrm{GPa}$ & $0.60 \mathrm{GPa}$ \\
\hline $\mathrm{CH} \cdots \mathrm{S}$ & $\begin{array}{l}2.804(7) / \\
3.712(9)\end{array}$ & $\begin{array}{l}2.830(3) / \\
3.706(3)\end{array}$ & $\begin{array}{l}2.873(9) / \\
3.692(9)\end{array}$ & $\begin{array}{l}2.889(7) / \\
3.723(7)\end{array}$ & $\begin{array}{l}2.762(5) / \\
3.680(5)\end{array}$ & $\begin{array}{l}2.763(3) \\
3.673(3)\end{array}$ \\
\hline $\mathrm{NH}^{\cdots}{ }^{\cdots \mathrm{Cl}}$ & $\begin{array}{l}2.364(3) / \\
3.176(10) \\
\end{array}$ & $\begin{array}{l}2.382(3) / \\
3.153(16) \\
\end{array}$ & $\begin{array}{l}2.461(5) / \\
3.231(14)\end{array}$ & $\begin{array}{l}2.452(4) / \\
3.220(12)\end{array}$ & $\begin{array}{l}2.437(4) / \\
3.197(10)\end{array}$ & $\begin{array}{l}2.443(2) \\
3.190(6) \\
\end{array}$ \\
\hline $\mathrm{CH}^{\cdots}{ }^{\cdots \mathrm{Cl}}$ & $\begin{array}{l}2.884(4) / \\
3.676(12)\end{array}$ & $\begin{array}{l}2.825(3) / \\
3.611(12)\end{array}$ & $\begin{array}{l}2.823(3) / \\
3.610(12)\end{array}$ & $\begin{array}{l}2.843(3) / \\
3.631(12)\end{array}$ & $\begin{array}{l}2.796(2) / \\
3.592(9)\end{array}$ & $\begin{array}{l}2.775(1) \\
3.567(6)\end{array}$ \\
\hline
\end{tabular}




\begin{tabular}{|l|l|l|l|l|l|l|}
\hline \multirow{2}{*}{$\mathrm{OH} \cdots \mathrm{Cl}$} & $2.295(8) /$ & $2.729(3) /$ & $2.502(5) /$ & $2.466(6) /$ & $2.362(4) /$ & $2.475(2) /$ \\
& $3.06(3)$ & $3.136(15)$ & $3.179(17)$ & $3.149(14)$ & $3.143(12)$ & $3.164(8)$ \\
\hline \multirow{2}{*}{$\cdots \cdots \mathrm{O}$} & $2.010(14) /$ & $1.934(8) /$ & $2.050(15) /$ & $1.989(11) /$ & $2.021(11) /$ & $2.068(8) /$ \\
& $2.836(17)$ & $2.765(14)$ & $2.865(18)$ & $2.818(16)$ & $2.822(15)$ & $2.855(10)$ \\
\hline
\end{tabular}

Table S2. The torsion angles for XylHCl hydrates $\mathrm{H} 1$ and $\mathrm{H} 2$.

\begin{tabular}{|l|l|l|l|l|l|l|}
\hline $\mathbf{X y l H C l} \cdot\left(\mathbf{C H}_{3}\right)_{2} \cdot \mathbf{H}_{2} \mathbf{O}$ & $0.12 \mathrm{GPa}$ & $0.19 \mathrm{GPa}$ & $0.23 \mathrm{GPa}$ & $0.34 \mathrm{GPa}$ & $0.41 \mathrm{GPa}$ & $0.60 \mathrm{GPa}$ \\
\hline $\mathrm{S}(1)-\mathrm{C}(2)-\mathrm{N}(7)-\mathrm{C}(8)$ & $-5(1)$ & $-11(3)$ & $-11(3)$ & $11(2)$ & $\begin{array}{l}1(2) \\
9(2)\end{array}$ & $\begin{array}{l}3(1) \\
11(1)\end{array}$ \\
\hline $\mathrm{N}(3)-\mathrm{C}(2)-\mathrm{N}(7)-\mathrm{C}(8)$ & $-2(2)$ & $-7(1)$ & $3(2)$ & $6(2)$ & $6(1)$ & $2(1)$ \\
& & & & & $13(2)$ & $11(1)$ \\
\hline $\mathrm{C}(2)-\mathrm{N}(7)-\mathrm{C}(8) \mathrm{C}(13)$ & $-93(1)$ & $-92(2)$ & $-95(2)$ & $-94(2)$ & $-95(1)$ & $\begin{array}{l}94(1) \\
91(1)\end{array}$ \\
\hline $\mathrm{C}(2)-\mathrm{N}(7)-\mathrm{C}(8)-\mathrm{C}(9)$ & $88(1)$ & $88(1)$ & $88(2)$ & $88(1)$ & $-87(2)$ & $-89(1)$ \\
& & & & & $-88(2)$ & $-90(1)$ \\
\hline
\end{tabular}

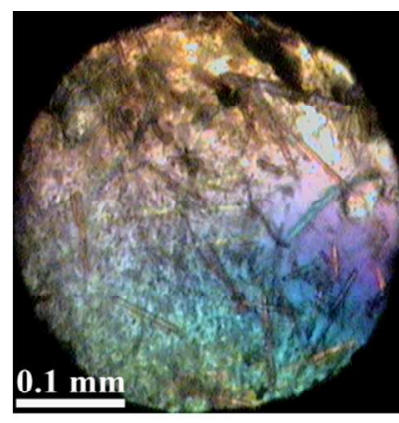

$339 \mathrm{~K}$

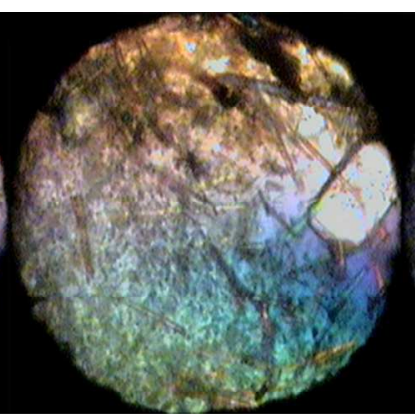

$320 \mathrm{~K}$

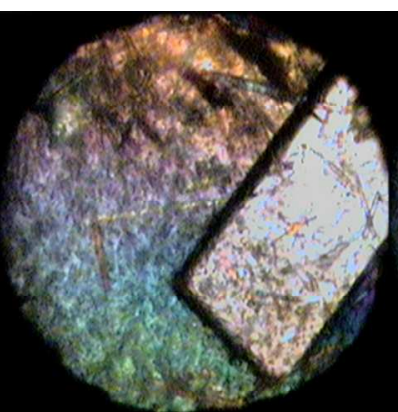

$316 \mathrm{~K}$

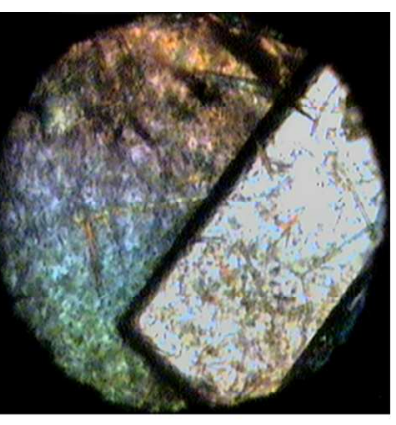

$303 \mathrm{~K}$

Figure S3. The single-crystal growth of the neat $\mathrm{XylHCl}$ form $\mathrm{Z}$ at $0.11 \mathrm{GPa}$.
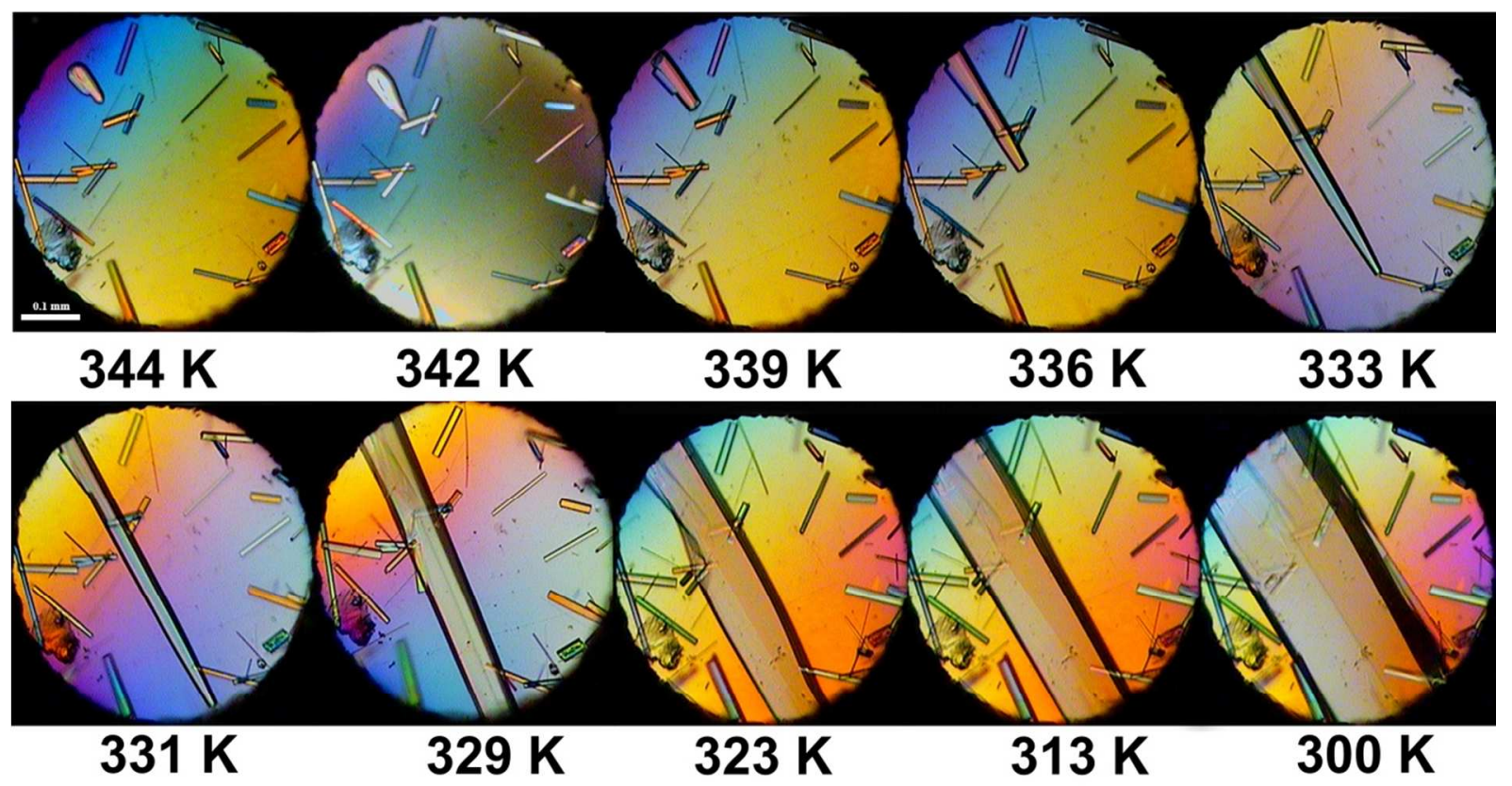

Figure S4. The single-crystal growth of the hydrate H1 form at $0.34 \mathrm{GPa}$. 


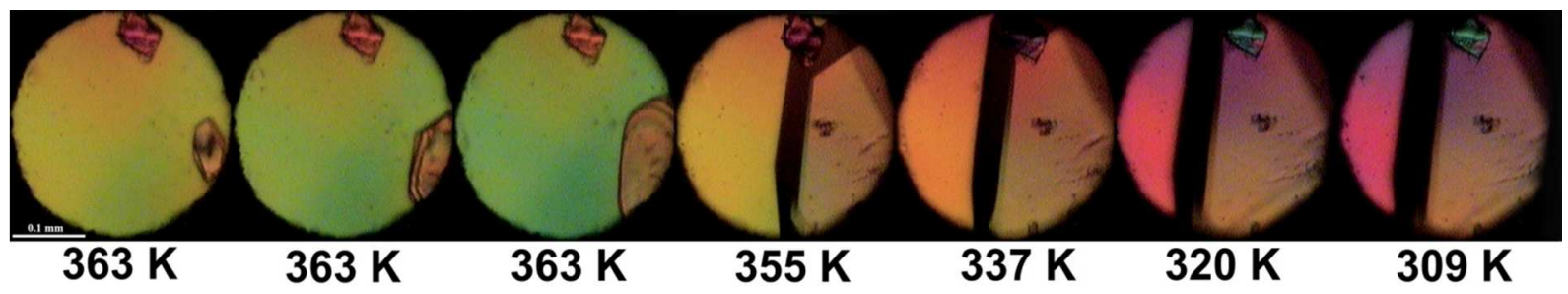

Figure S5. The single-crystal growth of the $\mathrm{CHCl}_{3}$ solvate at $0.21 \mathrm{GPa}$.

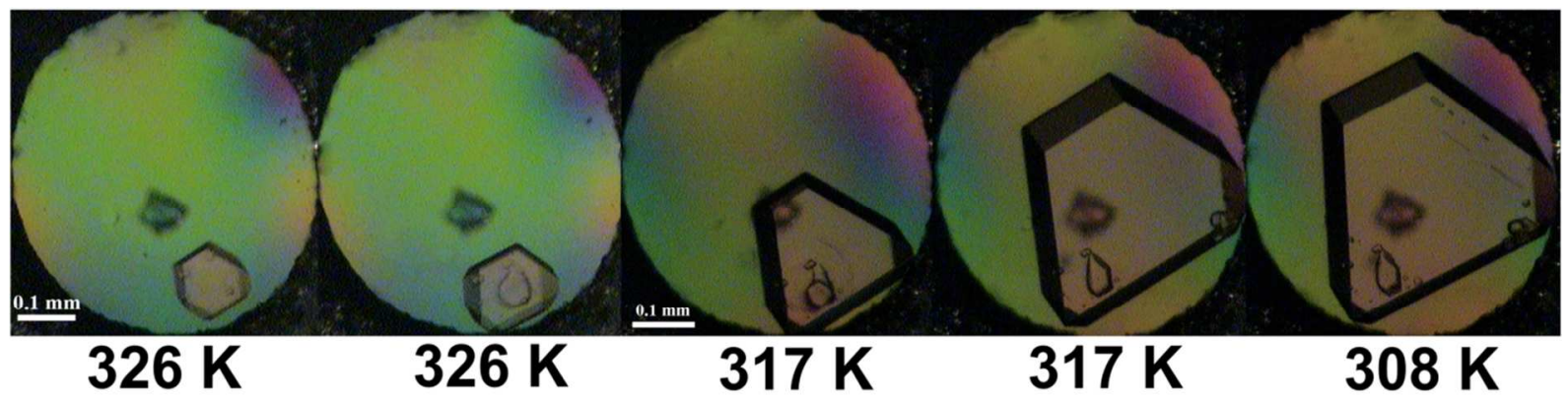

Figure S6. The single-crystal growth of the $\mathrm{CH}_{2} \mathrm{Cl}_{3}$ solvate at $0.13 \mathrm{GPa}$.

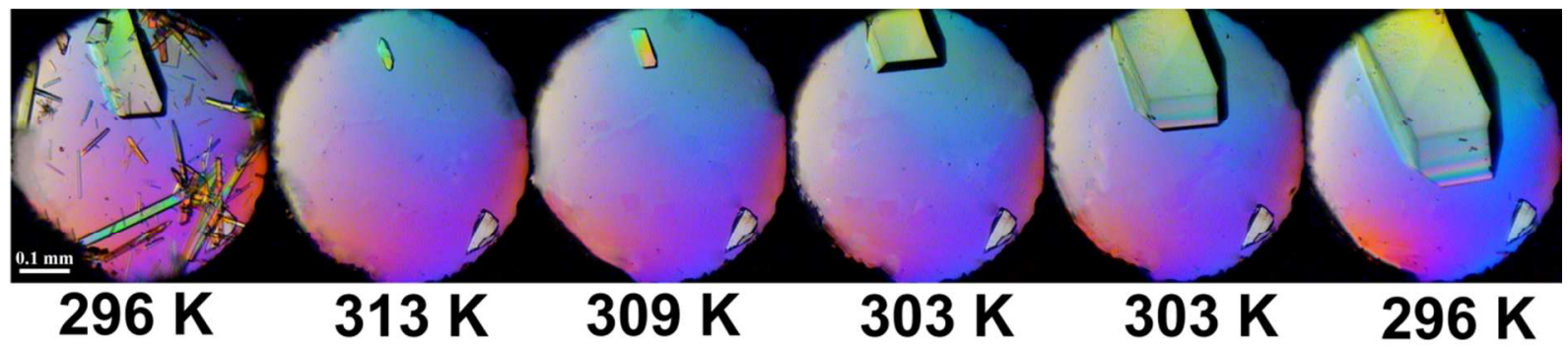

Figure S7. The single-crystal growth of the $\left(\mathrm{CH}_{3}\right)_{2} \mathrm{CHOH}$ solvate at $0.21 \mathrm{GPa}$. 

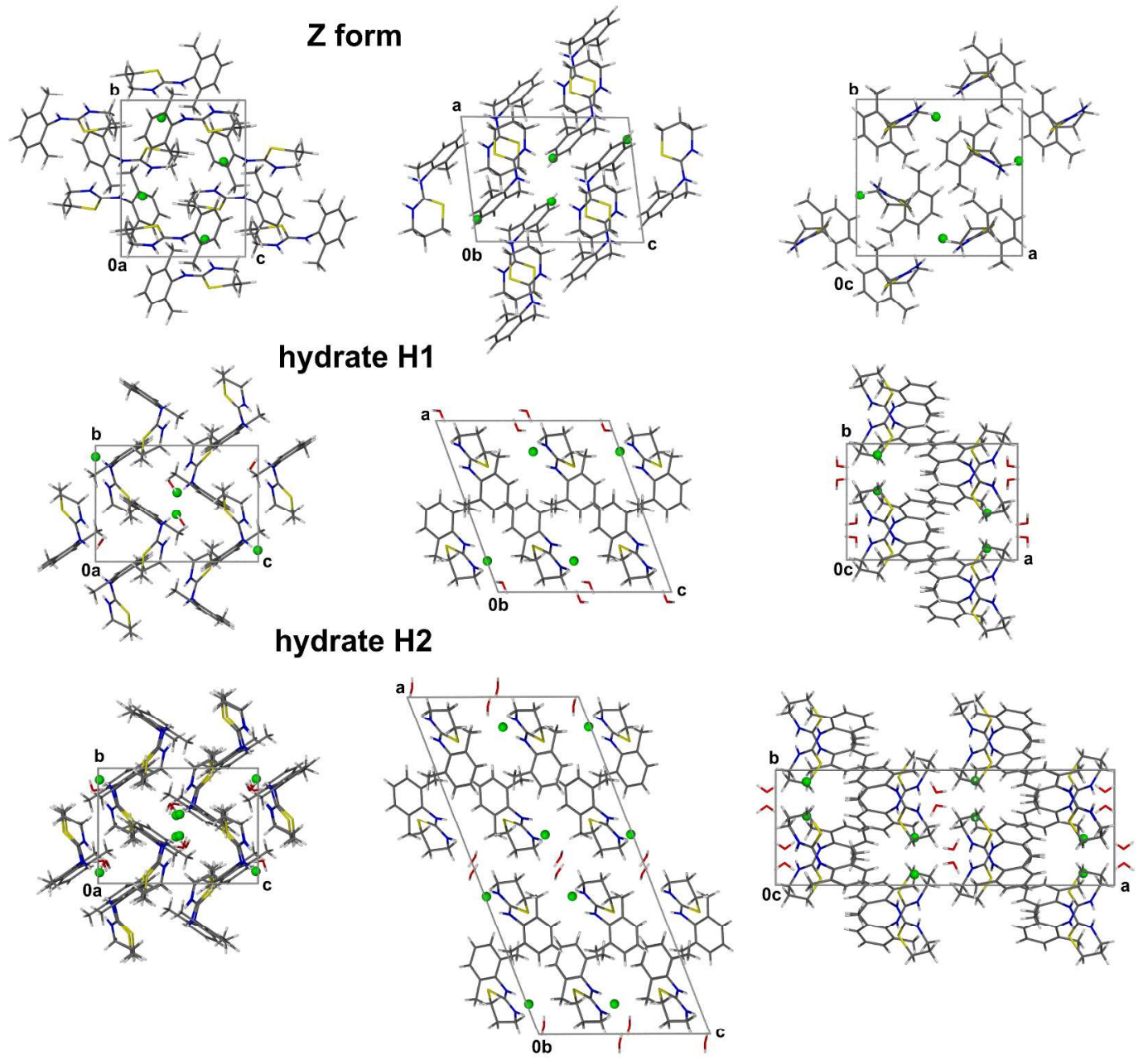

$\left(\mathrm{CH}_{3}\right)_{2} \mathrm{CH}_{2} \mathrm{OH}$ solvate
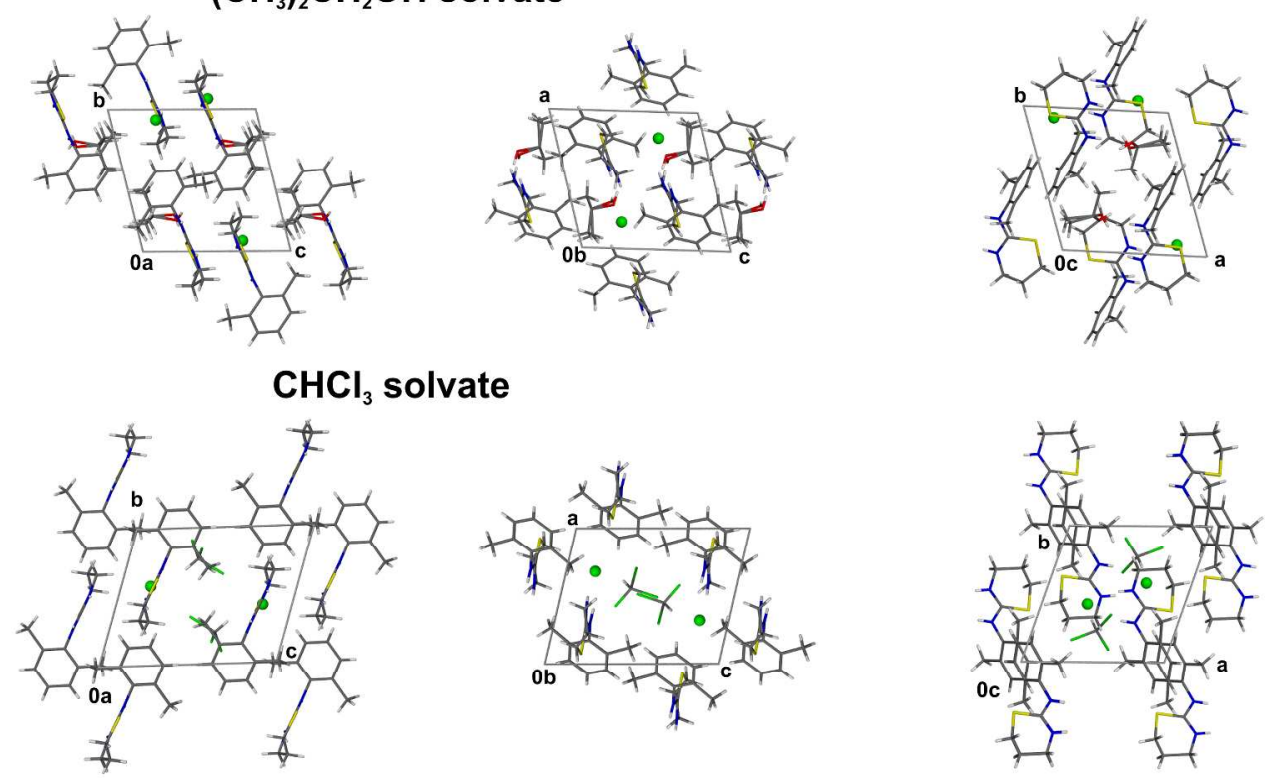

Figure S8. Unit-cell packing of $\mathrm{XylHCl}$ neat form and its solvates. 